\title{
LDL cholesterol levels in patients with coronary artery disease in real word: data from Cardiovascular Registry of Trieste
}

Antonella Cherubini*, Andrea Paolmba', Marco Morosin', Giulia Russo', Carmine Mazzone', Maurizio Fisicaro', Giulia Barbati', Kira Stellato', Gianni Cioffi', Luigi Tarantini', Giovanni Pulignano ${ }^{4}$, Luigi Cattin', Andrea Di Lenarda

${ }^{1}$ Cardiovascular Center, Health Authority $n^{\circ} 1$ and University of Trieste, Maggiore Hospital, Trieste, Italy ${ }^{2}$ Department of Cardiology, Villabianca Hospital, Trento, Italy

${ }^{3}$ Department of Cardiology, S.Martino Hospital, Belluno, Italy

${ }^{4}$ Cardiology Department n1, San Camillo Hospital, Rome, Italy

Background: From the literature we know that less than $50 \%$ of patients with coronary artery disease (CAD) reaches the target LDL cholesterol $<100 \mathrm{mg} / \mathrm{dl}$ in clinical practice. The 2012 European Guidelines on Prevention lowers the target to $<70 \mathrm{mg} / \mathrm{dl}$ in very high risk patients.

Methods: We analyzed the clinical data, levels of LDL cholesterol, the statins prescription and the medium term outcome in patients with chronic coronary artery disease in 5,106 patients with CAD enrolled from November 2009 to December 2012 in Cardiovascolar Registry of Trieste (CVRT). Clinical data were derived from the E-data chart for outpatient clinic (Cardionet $囚$ ) of Cardiovascular Center of Trieste, Italy.

Results: At the first clinical evaluation only $59.7 \%$ of patients with CAD had the level of LDL cholesterol available; they were younger (age $>75$ yo $42 \%$ vs $46 \%, p=0,002$ ), had more cardiovascular (CV) risk factors, comorbidities (Charlson index $>5$ ) and statins prescription ( $70 \%$ vs $61 \%$ ), but less frequent stroke or TIA. In the group with LDL available,

Received: $1^{\text {st }}$ May 2014

*Address for correspondence: Centro Cardiovascolare, Ospedale Maggiore, Via Slataper, 9, 34128 Trieste, Italy

E-mail: antonella.cherubini@ass1.sanita.fvg.it
$17 \%$ had LDL cholesterol $<70 \%$ and $53 \%$ LDL ct $<100$ $\mathrm{mg} / \mathrm{dl}$. The patients at target level of LDL cholesterol were more frequently males, with more frequent CV risk factors and history of CV events, more frequent comorbidities and $\geq 5$ drugs prescribed. The level of LDL cholesterol influenced the prescription of statins: in the group with LDL cholesterol $\geq 100 \mathrm{mg} / \mathrm{dl}$, the cardiologists started, increased the dosage or changed the statin therapy in more than twofold of cases, even if only in about $30 \%$ of cases. The group of patients at target LDL cholesterol on statin therapy had the best prognosis (survival free from death and/or hospitalization $75 \%$ at 3 years). On the other hand, the group of patients with low LDL cholesterol level not on statin therapy (older with more advanced CV and not CV disease) had the worst prognosis (survival free from death and/or hospitalization $45 \%$ at 3 years, $p<0.001$ ).

Conclusions: In our population of outpatients with CAD enrolled in CVRT, the target LDL cholesterol $<70 \mathrm{mg} / \mathrm{dl}$ and $<100 \mathrm{mg} / \mathrm{dl}$ was reached by $17 \%$ and $53 \%$ of cases. The availability and target level of LDL cholesterol influenced the statins prescription, but there is still a large room to improve proactive cardiology intervention in very high risk patiens. The medium term outcome is strongly related to the target

KEYWORDS: coronary heart disease, hyperlipemia, cholesterol, statin, secondary prevention.

CITATION: Cardiol Croat. 2014;9(5-6):247.

\section{Literature}

1. Kotseva K, Wood D, De Backer G, De Bacquer D, Pyörälä K, Keil U; EUROASPIRE Study Group. Cardiovascular prevention guidelines in daily practice: a comparison of EUROASPIRE I, II, and III surveys in eight European countries. Lancet. 2009;373(9667):929-40.

2. The Fifth Joint Task Force of the European Society of Cardiology and Other Societies on Cardiovascular Disease Prevention in Clinical Practice, European Guidelines on cardiovascular disease prevention in clinical practice (version 2012). Eur Heart J. 2012;33:1635-701. 